TITLE:

\title{
Nonlinear Koopman Modes and Power System Stability Assessment Without Models
}

\author{
$\operatorname{AUTHOR}(\mathrm{S})$ :
}

Susuki, Yoshihiko; Mezic, Igor

\section{CITATION:}

Susuki, Yoshihiko ...[et al]. Nonlinear Koopman Modes and Power System Stability Assessment Without Models. IEEE Transactions on Power Systems 2013, 54(2): 899-907

\section{ISSUE DATE:}

2013-11-06

URL:

http://hdl.handle.net/2433/179433

\section{RIGHT:}

(c) 2013 IEEE. Personal use of this material is permitted. Permission from IEEE must be obtained for all other uses, in any current or future media, including reprinting/republishing this material for advertising or promotional purposes, creating new collective works, for resale or redistribution to servers or lists, or reuse of any copyrighted component of this work in other works.; この論文は出版社版でありません。引用の際には出版社版をご確認ご利用ください。; This is not the published version. Please cite only the published version. 


\title{
Nonlinear Koopman Modes and Power System Stability Assessment without Models
}

\author{
Yoshihiko Susuki and Igor Mezić
}

\begin{abstract}
The loss of stability - an instability - can become a critical cause of emergent cascading outages leading to widespread blackouts. Penetration of renewable energy sources makes the problem of instability more urgent because of the highly fluctuating nature of such sources. Here we show a databased approach to stability assessment of power systems without models. This approach is enabled by Koopman mode analysis for nonlinear dynamical systems, which detects an instability based on the properties of the point spectrum of the Koopman operator. We apply the technique to data on physical power flows sampled from the two major accidents, the 2011 ArizonaSouthern California grid outage and the 2006 system disturbance of the European interconnected grid, and successfully detect unstable power flow patterns that govern the complex dynamics occurring during the accidents.
\end{abstract}

Index Terms-power system, stability assessment, Koopman mode, cascading outage

\section{INTRODUCTION}

C ASCADING outages leading to wide-spread blackouts in large-scale interconnected power systems are repeatedly emerging in the world. Examples of this include the 1965 Northeast America blackout, the 1996 West North America blackouts, and the 2003 blackouts in North America and Europe [1]. Cascading outage is a sequence of correlated outages of individual components that successively weaken the power system, leading to its collapse. Many groups of researchers have investigated the complex dynamics occurring in power systems: see [2]-[8] and [9] as a comprehensive review. Understanding the dynamics and maintaining the system-wide stability are great challenges of science and engineering.

Wider penetration of renewable energy sources has become a potential cause of power system instability. Renewable sources include solar and wind power generations, and their outputs normally fluctuate due to the uncertainty in weather. In the modern power system with a large number of distributed renewable sources, the fluctuating power sources lead to more instability. Many studies on this topics have been reported: see e.g. [10]. In the final report of the 2006 System Disturbance in the European Grid [11], it was suggested that the uncontrolled

This work was partially supported by Itoh Chubei Foundation to Y. Susuki, and by AFOSR grant FA9550-09-1-0141 and ARO grant W911NF-11-1-0511 to I. Mezić.

Y. Susuki is with the Department of Electrical Engineering at Kyoto University, Nishikyo, Kyoto 615-8510 Japan, and JST-CREST, 4-1-8 Honcho, Kawaguchi, Saitama 332-0012 Japan (e-mail: susuki@ieee.org).

I. Mezić is with Institute for Energy Efficiency, Center for Control, Dynamical Systems and Computation, and Department of Mechanical Engineering, University of California, Santa Barbara, CA 93106 (e-mail: mezic@engineering.ucsb.edu). operation of distributed sources (mainly wind and combinedheat-and-power) during the disturbance complicated the process of re-establishing normal system conditions. Thus, the penetration of renewable sources makes it urgent to develop methods for instability detection.

Many methods for stability assessment of power systems have been developed [12]-[14]. Traditional methods are mainly model-based, that is, the stability assessment is performed by investigating a mathematical model that represents the target dynamics of a power system. It is widely recognized that cascading outages are fairly complicated emergent phenomena in the high-dimensional nonlinear dynamical systems. Therefore, it is difficult to obtain a mathematical model that explains all events and time evolution of a cascading outage. Even if we could obtain such a model, it would be not easy to gain a dynamical insight to the cascading outages from the model, because of its complexity. Also, the large increase of renewable sources makes it hard to obtain a relevant deterministic model because of their uncertain nature. Thus, in contrast to the model-based approach, it is necessary to develop methods that indicate spatio-temporal structure of instability phenomena and their precursors from data. Standard SCADA (Supervisory Control And Data Acquisition) systems continuously collect information of the system's state and distribute it to system operators. Recent advance of real-time PMUs (Phasor Measurement Units) offers an advanced data collection method using phases of AC voltages: see e.g. [15][17]. Several reports exist on this line of research [18]-[20]. In this paper, we present an approach to stability assessment based on measured physical power flow data. In contrast to the existing methods, our approach provides not only dynamic patterns of power flows, which we refer to as the base flow patterns below, but also stability information. Our approach is conducive to development of new methods for monitoring and control of large-scale, emergent events in power systems and enables operational techniques such as situational awareness. This is catalyzed by developments in theory of nonlinear dynamical systems using Koopman Mode Analysis (KMA), based on analysis of properties of the point spectrum of the Koopman operator [21]-[25].

The rest of this paper is organized as follows. In Section II we describe the KMA for analysis of finite-time data on dynamics of physical power flows and propose a KMAbased approach to stability assessment of power systems. In Sections III and IV we apply the technique to data sampled in the two major accidents, the 2011 Arizona-Southern California grid outage and the 2006 system disturbance of the European interconnected grid. Conclusions of this paper are presented 
in Section V.

\section{Stability Assessment Via Nonlinear Koopman Mode ANALYsis}

Consider the finite-time data on dynamics of physical power flows (more precisely, active power flows) under uniform sampling, given by

$$
\left\{\boldsymbol{P}_{0}, \boldsymbol{P}_{1}, \ldots, \boldsymbol{P}_{N-1}\right\},
$$

where $\boldsymbol{P}_{k} \in \mathbb{R}^{m}$ is the snapshot of power flows at the discrete time $k, m$ the number of measurement sites (for example, generation plants, transmission lines, and substations), and $N$ the number of available snapshots. Generally speaking, the power flows are determined by the internal states of a system such as rotating frequencies and voltages of $\mathrm{AC}$ generators in power plants, bus voltages in substations, power consumptions in loads, and states of controllers in plants and substations [13]. Here we use $\boldsymbol{x}$ to represent all the internal states belonging to a space $\mathbb{X}$ and assume that the time evolution of $\boldsymbol{x}$ is represented by the deterministic, nonlinear dynamical system as follows:

$$
\boldsymbol{x}_{k+1}=\boldsymbol{T}\left(\boldsymbol{x}_{k}\right), \quad k=0,1, \ldots
$$

where $\boldsymbol{T}: \mathbb{X} \rightarrow \mathbb{X}$ is a nonlinear vector-valued map. The map describes the rule of how $\boldsymbol{x}$ evolves in time. If the map is constructed via power system modeling techniques (see e.g. [13]), then the dimension of the map is possibly very large. Now, in order to associate the finite-time data with the internal dynamics, we define a vector-valued map defined on $\mathbb{X}$, which we refer to as an observable. For the current analysis, it is reasonable to define the observable as a map $\boldsymbol{f}_{\mathrm{P}}: \mathbb{X} \rightarrow \mathbb{R}^{m}$ such that the snapshot $\boldsymbol{P}_{k}$ of power flows at time $k$ is written as

$$
\boldsymbol{P}_{k}=\boldsymbol{f}_{\mathrm{P}}\left(\boldsymbol{x}_{k}\right) .
$$

Mathematically, the time evolution of an observable is modeled by the so-called Koopman operator [26] that is defined for the underlying dynamical system (2). The Koopman operator is a linear operator $\mathcal{U}$ acting on scalar-valued functions on $\mathbb{X}$ : for a function $f: \mathbb{X} \rightarrow \mathbb{C}, \mathcal{U}$ maps $f$ into a new function $\mathcal{U} f$, given by

$$
(\mathcal{U} f)(\boldsymbol{x}):=f(\boldsymbol{T}(\boldsymbol{x})) .
$$

Although the dynamical system (2) is nonlinear and evolves on a finite-dimensional space, the Koopman operator $\mathcal{U}$ is linear but infinite-dimensional. Such a linear operator defined for arbitrary nonlinear dynamical systems [27], [28] captures the full information of the nonlinear dynamics of the system (2), that is, the internal dynamics of a system. The eigenfunctions and eigenvalues of $\mathcal{U}$ are defined as follows: for $\varphi_{j}: \mathbb{X} \rightarrow \mathbb{C}$ and constants $\lambda_{j} \in \mathbb{C}$, such that

$$
\mathcal{U} \varphi_{j}(\boldsymbol{x})=\lambda_{j} \varphi_{j}(\boldsymbol{x}), \quad j=1,2, \ldots
$$

the functions $\varphi_{j}$ are referred to as Koopman eigenfunctions, and the constants $\lambda_{j}$ as the associated Koopman eigenvalues. The set of all Koopman eigenvalues is called the point spectrum of the Koopman operator [22]. See [21], [22], [24], [25] for examples that explain the essence of the mathematical background.
The idea which we now propose for power system stability assessment is to investigate the finite-time data on power flows in terms of the Koopman eigenfunctions and Koopman eigenvalues. Here we develop the analysis for the case when there are unstable eigenvalues of the Koopman operator, but we have access to data for only a short period of time. We call the analysis based on the point spectrum of the Koopman operator the Koopman Mode Analysis (KMA). The outline of the KMA is as follows. If each of the $m$ components of $\boldsymbol{f}_{\mathrm{P}}$ lies within the span of eigenfunctions $\varphi_{j}$, then the vector-valued function $f_{\mathrm{P}}$ is expanded in terms of these eigenfuntions as

$$
\boldsymbol{f}_{\mathrm{P}}(\boldsymbol{x})=\sum_{j=1}^{\infty} \varphi_{j}(\boldsymbol{x}) \boldsymbol{V}_{j},
$$

where $\boldsymbol{V}_{j} \in \mathbb{C}^{m}$ are regarded as the vector coefficients in the expansion. Thus, the time evolution $\boldsymbol{f}_{\mathrm{P}}\left(\boldsymbol{x}_{k}\right)$ starting at $\boldsymbol{f}_{\mathrm{P}}\left(\boldsymbol{x}_{0}\right)$ is represented as

$$
\boldsymbol{f}_{\mathrm{P}}\left(\boldsymbol{x}_{k}\right)=\sum_{j=1}^{\infty} \varphi_{j}\left(\boldsymbol{x}_{k}\right) \boldsymbol{V}_{j}=\sum_{j=1}^{\infty} \lambda_{j}^{k} \varphi_{j}\left(\boldsymbol{x}_{0}\right) \boldsymbol{V}_{j},
$$

i.e.,

$$
\boldsymbol{P}_{k}=\sum_{j=1}^{\infty} \lambda_{j}^{k} \varphi_{j}\left(\boldsymbol{x}_{0}\right) \boldsymbol{V}_{j} .
$$

Thus, we refer to $\boldsymbol{V}_{j}$ as the Koopman Mode (KM) associated with $\lambda_{j}$. If the dynamics have only a finite number of discrete spectra (peaks) in complex plane, then the expansion (8) gives a good approximation of the dynamics. The Koopman eigenvalue $\lambda_{j}$ characterizes the temporal behavior of the corresponding $\mathrm{KM} \boldsymbol{V}_{j}$ : the phase of $\lambda_{j}$ determines its frequency, and its modulus determines the growth rate. An unstable Koopman eigenvalue is an eigenvalue whose modulus is larger than one, corresponding to a KM that grows exponentially as time increases. We propose the following method for detection of power system instability: if for a given set of data on power flows obtained over a time interval, there exists no unstable Koopman eigenvalue, then we conclude that the associated power system is stable. If not, the power system behaves in an unstable manner.

Here we discuss the notion of stability addressed in this paper. Our analysis is based on data for only a short period of time, in other words, is intended to describe short-term dynamics exhibited by a (possibly) nonlinear dynamical system. In this sense, the current notion of stability is different from the conventional one that is intended to long-term, asymptotic dynamics. In a dynamical viewpoint, the current notion deals with a portion of a trajectory and is formulated by examining the unstable eigenvalues of the finite-time Koopman operator corresponding to the time period over which the trajectory is executed. The difference of notions of stability can be seen by considering a periodic orbit that slows down as it passes near a saddle and then speeds up. Portions of such an orbit could be judged stable or unstable by the method of the paper depending on which portion is chosen. In this paper, we intend to provide a new method for detecting such a short-term trend in dynamics from observational data, thereby obtaining an insight to control measures for (nearly) real-time management. 
Computation of Koopman eigenvalues and modes is a challenging problem. In [23], the Arnoldi-like algorithm for computing the Koopman eigenvalues and modes was developed. The algorithm has been widely used for analysis of fluid flow models (see e.g. [23], [25]), power system simulation data [29], [30], and building system data [31]. In particular, in [29], [30] the algorithm successfully captures nonlinear oscillations emerging in power systems. However, in this series of applications, it has not been used to detect instabilities from data. In our approach, the Koopman eigenvalues $\lambda_{j}$ are approximately computed as the empirical Ritz values $\tilde{\lambda}_{j} \in \mathbb{C}$, and the terms $\varphi_{j}\left(\boldsymbol{x}_{0}\right) \boldsymbol{V}_{j}$ of Koopman eigenfunctions and modes as the empirical Ritz vectors $\tilde{\boldsymbol{V}}_{j} \in \mathbb{C}^{m}$ (see Appendix A).

We use the above approach to detecting instabilities based on KMA to analyze data on power flows sampled from the two major accidents: the 2011 Arizona-Southern California grid outage and the 2006 system disturbance of the European interconnected grid. Our main idea of the applications is to decompose the data on power flows into a set of KMs that imply base flow patterns. A base flow pattern is geographically distributed over the system, exhibits power flows that evolve coherent oscillatory or exponential (growing or decaying) behavior, and is regarded as a coherent spatial unit of power flows. Let $\tilde{\boldsymbol{V}}_{j}=\left[\tilde{V}_{j 1}, \tilde{V}_{j 2}, \ldots, \tilde{V}_{j m}\right]^{\mathrm{T}}$ denote the $j$ th empirical Ritz vector associated with the possibly complexvalued Ritz value $\tilde{\lambda}_{j}=\tilde{r}_{j} \exp \left(\mathrm{i} 2 \pi \tilde{\nu}_{j}\right)$ for the finite-time data. For convenience we will call the empirical Ritz vector $\tilde{V}_{j}$ the KM, although it differs from it possibly by a constant. The symbol $\mathrm{T}$ represents the transpose operation of vectors, and $\mathrm{i}$ the imaginary unit. Since the original data are real-valued, if the $\mathrm{KM} \tilde{\boldsymbol{V}}_{j}$ is complex-valued, then there exists the conjugate $\mathrm{KM} \tilde{\boldsymbol{V}}_{j}^{\mathrm{c}}$ with the conjugate Ritz value $\tilde{\lambda}_{j}^{\mathrm{c}}$ in the expansion (8), where $\mathrm{c}$ represents the complex conjugate operation of scalars and vectors. Here we suppose that the $(j+1)$-th $\mathrm{KM} \tilde{\boldsymbol{V}}_{j+1}$ corresponds to the conjugate vector $\tilde{\boldsymbol{V}}_{j}^{\mathrm{c}}$ and the $(j+1)$-th Ritz value $\tilde{\lambda}_{j+1}$ to $\tilde{\lambda}_{j}^{\mathrm{c}}$. In this way, the dynamics of base flow pattern described by the conjugate pair of KMs, $\boldsymbol{P}_{k}^{\{j, j+1\}}$, are given as

$$
\begin{aligned}
\boldsymbol{P}_{k}^{\{j, j+1\}}= & \tilde{\lambda}_{j}^{k} \tilde{\boldsymbol{V}}_{j}+\left(\tilde{\lambda}_{j+1}\right)^{k} \tilde{\boldsymbol{V}}_{j+1} \\
= & 2 \tilde{r}_{j}^{k}\left[\begin{array}{c}
\left|\tilde{V}_{j 1}\right| \cos \left\{2 \pi k \tilde{\nu}_{j}+\operatorname{Arg}\left(\tilde{V}_{j 1}\right)\right\} \\
\left|\tilde{V}_{j 2}\right| \cos \left\{2 \pi k \tilde{\nu}_{j}+\operatorname{Arg}\left(\tilde{V}_{j 2}\right)\right\} \\
\vdots \\
\left|\tilde{V}_{j m}\right| \cos \left\{2 \pi k \tilde{\nu}_{j}+\operatorname{Arg}\left(\tilde{V}_{j m}\right)\right\}
\end{array}\right],
\end{aligned}
$$

where $k=0,1, \ldots, N-2,\left|\tilde{V}_{j p}\right|$ denotes the modulus of the $p$ th element $\tilde{V}_{j p}$ of $\tilde{\boldsymbol{V}}_{j}$, and $\operatorname{Arg}\left(\tilde{V}_{j p}\right)$ does the argument of $\tilde{V}_{j p}$. The spatial shape of the dynamics is captured by the modulus and argument of the KM. In the following, we investigate the dynamics of power flows by directly computing the KMs from measured data.

Here note that an exponential approximation of data similar to (9) is obtained with the well-known Prony analysis [32], [33]. The technique is thought of as a fit, but in fact the Koopman operator theory shows that many dynamical systems can be analyzed exactly in those terms. The difference of the two methods is in our case of computational methods developed specifically for KMA and in their justification by dynamical systems theory.

\section{2011 ARIZONA-SOUTHERN CALIFORNIA GRID OUTAGE}

The outage occurred in the afternoon of September 8, 2011, affected parts of Arizona, Southern California, and Baja California (in Mexico), and left approximately 2.7 million customers without power [34]. The grid includes the four areas of main power consumption, which are denoted by San Diego, IID (Imperial Irrigation Distinct) north/south, and Yuma Pocket, and their interconnection via substations and AC transmission lines with different voltage levels. These four areas were ultimately islanded (no import of electricity and consequently no electricity inside of them).

The data on power flows recorded with SCADA and PMU systems are available in the official report [34], and the following analysis depends on the report. One-line diagram of the San Diego area power system given in Appendix B shows the location where data were collected.

Figure 1A shows the dynamics of power flows recorded at one interface and seven lines via SCADA, except for the Miguel-IV $500 \mathrm{kV}$ line for which the data were obtained via PMU. The data were obtained by sampling values from the original figures in Appendix D of [34] and applying to them the cubic interpolation under the uniform sampling (period $T=35 \mathrm{~s}$ ). The value of power flow on the Path $44500 \mathrm{kV}$ line (solid blue line) is divided by 10 in order to plot all the data in the same scale. Since the electric transients are sufficiently fast, the dynamics of power flows are relatively static and change slowly with time. In Fig. 1A, several flows go down to zero. This implies that the corresponding facilities are tripped. Other flows increased gradually because of the heavy loading conditions caused by the trip. These flow dynamics drove the grid's state to a severe situation, and finally at 15:38:02.4 just one line (Path $44500 \mathrm{kV}$ line) kept the power transfer to the central load in San Diego area. After 15:38:21.2 (immediately after the last time shown in Fig. 1A), the entire San Diego/IID/Yuma Pocket area power grid collapsed. No dominant power flow appeared in the aftermath.

We now apply the KMA to analyze the Arizona-Southern California grid outage using the data on power flows presented in Fig. 1A. The data correspond to the nine $(N=9)$ samples $\left\{\boldsymbol{P}_{0}, \boldsymbol{P}_{1}, \ldots, \boldsymbol{P}_{8}\right\}$ at eight different $(m=8)$ lines. In this setting, we computed the eight empirical Ritz values $\tilde{\lambda}_{j}$ and vectors $\tilde{\boldsymbol{V}}_{j}$ for the data and show the results in Figs. 1B and 1C. The corresponding values are summarized in Tab. I. The color of empirical Ritz values in Fig. 1B varies smoothly from red to white, depending on the norm of the corresponding vector, $\left|\tilde{\boldsymbol{V}}_{j}\right|$, except for mode 4 which denoted by a blue circle. Mode 4 is the average power flow associated with the Koopman eigenvalue $\tilde{\lambda}_{4} \approx 1$. In Fig. $1 \mathrm{~B}$, the three conjugate pairs of $\tilde{\lambda}_{j}$, labeled $\{1,2\},\{5,6\}$ and $\{7,8\}$, exist outside of the unit circle. The magnitude of the associated dynamical components grows over time, and thus they represent unstable dynamics. Mode $\{1,2\}$ has the fastest oscillation frequency of those. 


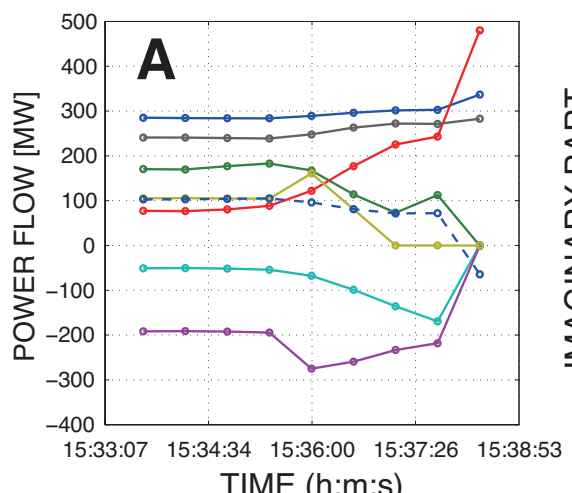

TIME (h:m:s)

$\rightarrow$ Path 44 500kV (x 1/10)

$\rightarrow$ IV-El Centro 230kV "S" line

$\rightarrow$ Miguel-IV 500kV line

$\rightarrow$ El Centro-Pilot Knob 161kV line

$\rightarrow$ Pilot Knob-Knob $161 \mathrm{kV}$ line

$\rightarrow$ Pilot Knob-Yucca 161kV "AX" line

$\rightarrow$ J Hinds-Mirage 230kV line

$\rightarrow$ - J Hinds-Eagle Mt. 230kV line

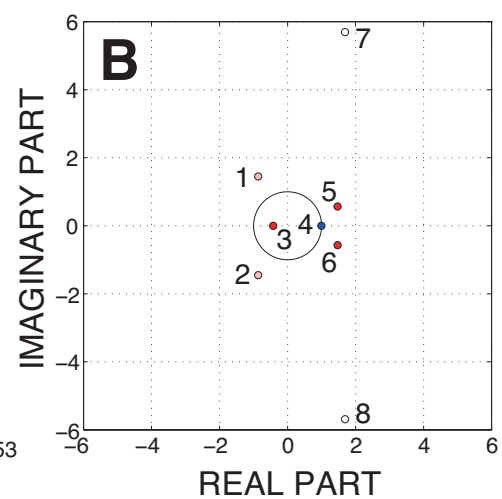

REAL PART

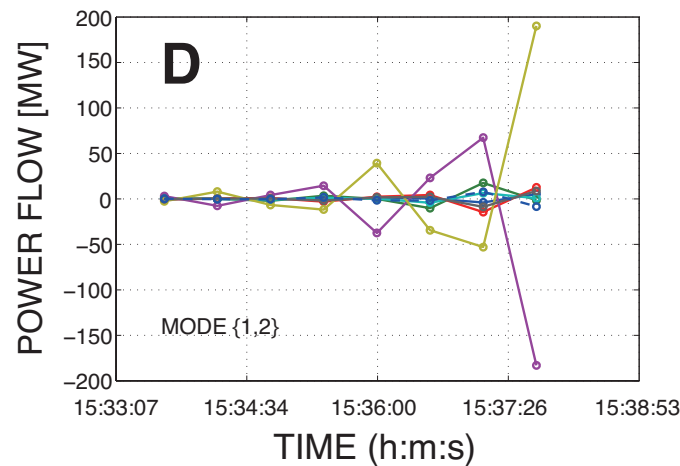

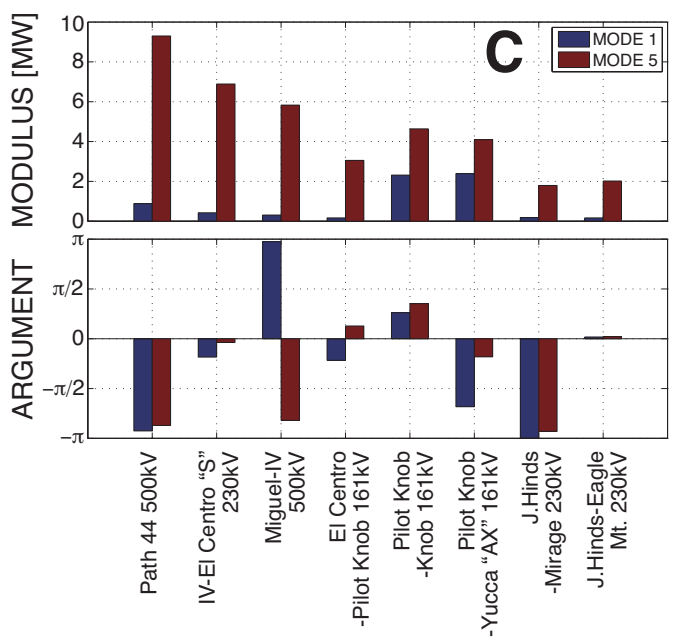

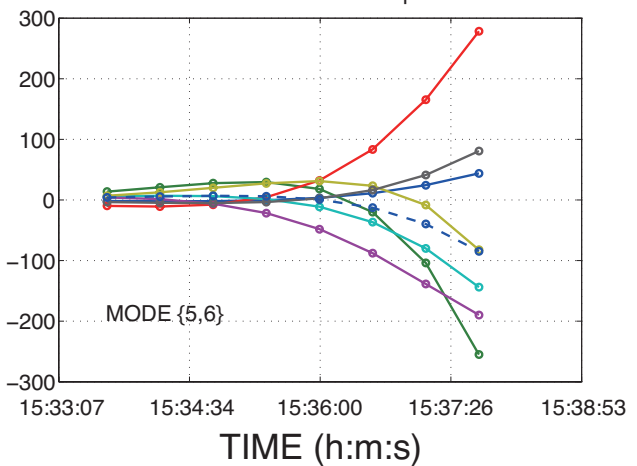

Fig. 1. Koopman mode analysis of power flow in the 2011 Arizona-Southern California grid outage: (A) power flow data; (B) Koopman eigenvalues $\tilde{\lambda}_{j}$; (C) modulus and argument vectors of Koopman modes $\tilde{\boldsymbol{V}}_{j}$ (see the main text for definitions); and (D) dynamics of base flow patterns (9). In the figures (A) and (D), the value of power flow on the Path $44500 \mathrm{kV}$ line is divided by 10 to plot all the data in one figure. Figure $\mathrm{C}$ shows $\tilde{\boldsymbol{V}}_{1}$ and $\tilde{\boldsymbol{V}}_{5}$, and Figure D shows the two unstable modes $\{1,2\}$ and $\{5,6\}$.

TABLE I

EMPIRICAL RITZ VALUES AND VECTORS FOR POWER FLOW IN THE 2011 ARIZONA-SOUTHERN CALIFORNIA GRID OUTAGE

\begin{tabular}{llclc}
\hline$j$ & $\left|\tilde{\boldsymbol{V}}_{j}\right|$ & $\tilde{\lambda}_{j}$ & $\left|\tilde{\lambda}_{j}\right|$ & Period [s] \\
\hline 1 & 3.5 & $-8.7 \times 10^{-1}+\mathrm{i} 1.5$ & 1.7 & $1.0 \times 10^{2}$ \\
2 & 3.5 & $-8.7 \times 10^{-1}-\mathrm{i} 1.5$ & 1.7 & $1.0 \times 10^{2}$ \\
3 & $1.6 \times 10$ & $-4.2 \times 10^{-1}$ & $4.2 \times 10^{-1}$ & $7.0 \times 10^{1}$ \\
4 & $2.9 \times 10^{3}$ & $9.98 \times 10^{-1}$ & $9.98 \times 10^{-1}$ & - \\
5 & $1.5 \times 10$ & $1.5+\mathrm{i} 5.6 \times 10^{-1}$ & 1.6 & $6.0 \times 10^{2}$ \\
6 & $1.5 \times 10$ & $1.5-\mathrm{i} 5.6 \times 10^{-1}$ & 1.6 & $6.0 \times 10^{2}$ \\
7 & $1.1 \times 10^{-3}$ & $1.7+\mathrm{i} 5.7$ & 5.9 & $1.7 \times 10^{2}$ \\
8 & $1.1 \times 10^{-3}$ & $1.7-\mathrm{i} 5.7$ & 5.9 & $1.7 \times 10^{2}$ \\
\hline
\end{tabular}

Mode $\{5,6\}$ is of the biggest magnitude (norm of the KM) among the unstable modes, while mode $\{7,8\}$ has a small KM magnitude and we will neglect it from this point on. Mode 3 in Fig. 1B is inside of the unit circle and represents stable dynamics. Some of the KMs $\tilde{\boldsymbol{V}}_{j}$ are shown in Fig. 1C: the modulus vector for $\tilde{\boldsymbol{V}}_{j}$ is defined as $\left[\left|\tilde{V}_{j 1}\right|,\left|\tilde{V}_{j 2}\right|, \ldots,\left|\tilde{V}_{j 8}\right|\right]^{\mathrm{T}}$ and the argument vector as $\left[\operatorname{Arg}\left(\tilde{V}_{j 1}\right), \operatorname{Arg}\left(\tilde{V}_{j 2}\right), \ldots, \operatorname{Arg}\left(\tilde{V}_{j 8}\right)\right]^{\mathrm{T}}$. In Fig. 1C, the name of transmission lines is stated along the horizontal axis for clarity. The dynamics of base flow patterns for the two unstable modes are shown in Fig. 1D.

Now, we discuss the dynamics of mode $\{1,2\}$ with reference to power grid dynamics described in [34]. The period of oscillation of mode $\{1,2\}$ is $100 \mathrm{sec}$, and the spatial shape of $\left|\tilde{\boldsymbol{V}}_{1,2}\right|$ has two peaks on the Pilot Knob-Knob line and the Pilot Knob-Yucca "AX" line. This mode is relatively fast compared with the other modes except for the stable one (see Tab. I). In the official report [34], the events of disturbances and trips of the two lines are categorized in Phase 5 "Yuma Load Pocket Separates from IID and WALC (Western Area Power Administration-Lower Colorado)" from 15:35:40 to 15:37:55. The Yuma area is connected to the IID south area via the $92 \mathrm{kV} / 161 \mathrm{kV}$ Pilot Knob substation. The Yuma area is also connected to the WALC's Gila $161 \mathrm{kV} / 69 \mathrm{kV}$ substation. In the beginning of Phase 5 at 15:35:40, the Gila $161 \mathrm{kV} / 69 \mathrm{kV}$ transformers were tripped. This caused temporal heavy flows on the Pilot Knob-Knob line and the Pilot Knob-Yucca "AX" line in order to keep the transmission of power to Yuma: we see this temporal trend in Fig.1A. The power went from the Knob substation, through the Pilot Knob substation, to the Yucca $161 \mathrm{kV} / 69 \mathrm{kV}$ substation. After this, the "AX" line was tripped at about 15:36:40, and one minute later (all or some of) Pilot Knob transformers were tripped. The occurrence of temporal heavy flows is captured well by the modulus and argument of mode $\{1,2\}$ in Fig. $1 \mathrm{C}$ and by the dynamics of base flow patterns in Fig. 1D, where the flows on the two lines oscillate in an anti-phase manner. This anti-phase in the base flow implies that it oscillates between Knob and Yucca via Pilot Knob. Thus, we conclude the fast unstable mode $\{1,2\}$ 
is associated with the destructive event observed in Phase 5.

Next, we investigate the dynamical meaning of mode $\{5,6\}$. The spatial shape of $\left|\tilde{\boldsymbol{V}}_{5,6}\right|$ implies that this mode involves all the lines in the slow-growing instability with the period of $600 \mathrm{sec}$. As in the official report [34], the instability emerges in the phenomenon observed in Phase 6 "High-Speed Cascade, Operation of the SONGS (Sa Onofre Nuclear Generating Station) Separation Scheme and Islanding of San Diego, IID, CFE, and Yuma" from 15:37:55 to 15:38:21.2. During Phase 6, the coarse power flow in the San Diego area power grid rotated in a counter-clockwise manner. The flow motion is captured well by dominant modulus and argument of mode $\{5,6\}$, in which the Path 44, Miguel-IV (Imperial Valley), and J. Hinds-Mirage lines have almost the same value of argument. The flows on the three lines increase coherently as shown in Figs. 1A and 1D. This excess loading, in particular, on the Path 44 line initiated the SONGS Separation Scheme that led to the loss of SONGS nuclear plants. The loss of the SONGS nuclear plants resulted in the complete blackout of the San Diego area power grid. Thus, the slow mode $\{5,6\}$ is associated with the slow-growing instability of the entire grid. From the analysis above, it is clear that the two unstable KMs, $\{1,2\}$ and $\{5,6\}$, which are extracted from data using KMA, are closely connected to major phases of the Arizona-Southern California grid outage.

\section{2006 SYSTEM DISTURBANCE IN THE EUROPEAN GRID}

This disturbance was recorded in the night of November 4, 2006 and affected the UCTE's (Union for the Co-ordination of the Transmission of Electricity's) synchronously European interconnected grid [11]. It begun in the North German transmission grid, led to a splitting of the UCTE grid into three areas, and caused an interruption of power supply for more than 15 millions European households. This splitting is due to the tripping of interconnected $\mathrm{AC}$ lines and implies that the three areas were asynchronously operated. In each of the areas a significant imbalance of power emerged.

The data on dynamics of power flows in the UCTE grid are introduced in Fig. 2A, where we show the dynamics of power exchange deviations from 21:50 till 22:10. A power exchange deviation is defined as the difference between the actual power exchanges and the scheduled power exchanges with other countries. The areas focused in this figure are Belgium, Switzerland, D-RWE, Spain, France, Italy, Netherland, and Portugal. In this sense, the current application is slightly different from that to the 2011 Arizona-Southern California grid outage, in which the direct power flow data were used. Below, we will demonstrate that our approach is capable of obtaining instability information from such different dynamic data sets. The data in Fig. 2A were obtained by sampling values from the original figure in Appendix 7 of [11] under the uniform sampling (period $T=30 \mathrm{~s}$ ). The final report [11] states, "These transient deviations are the result of a global shift in physical power flows within the UCTE synchronous grid area to changes in generation programs and exchange programs around 22:00. These curves show a situation which is rather normal and typical at that time." However, the next single operation (coupling of busbars in the Landesbergen substation in Germany at 22:10) after the transient deviations in Fig. 2A initiated the widespread disturbance in the entire European grid. The operation of coupling the busbars was intended to resolve the heavy power flow on the LandesbergenWehrendorf $380 \mathrm{kV}$ line in Germany, but it resulted in its immediate trip and initiated a cascade of line trips, starting with the $220 \mathrm{kV}$ Bielefeld/Ost-Spexard line and continuing within the E.ON Netz grid [11]. Therefore, the N-1 criterion ${ }^{1}$ of secure operation of the grid was not fulfilled before the busbar coupling [11].

We performed the KMA of the data on power exchange deviations presented in Fig. 2A. The data are the forty-one $(N=41)$ samples $\left\{\boldsymbol{P}_{0}, \boldsymbol{P}_{1}, \ldots, \boldsymbol{P}_{40}\right\}$ at eight different $(m=$ 8 ) countries. By the similar method as in the Arizona-Southern California case, the forty pairs of Koopman eigenvalues and modes $\tilde{\lambda}_{j}$ and $\tilde{\boldsymbol{V}}_{j}$ were computed and shown in Figs. $2 \mathrm{~B}$ and $2 \mathrm{C}^{2}$. In Fig. $2 \mathrm{~B}$, we see that one conjugate pair of $\tilde{\lambda}_{j}$ is located outside of the unit circle, and the corresponding vector represents the unstable mode. The other $\tilde{\lambda}_{j}$ 's are located inside of the unit circle, that is, they are associated with the stable modes. The modulus vectors $\left[\left|\tilde{V}_{j 1}\right|,\left|\tilde{V}_{j 2}\right|, \ldots,\left|\tilde{V}_{j 8}\right|\right]^{\mathrm{T}}$ and argument vectors $\left[\operatorname{Arg}\left(\tilde{V}_{j 1}\right), \operatorname{Arg}\left(\tilde{V}_{j 2}\right), \ldots, \operatorname{Arg}\left(\tilde{V}_{j 8}\right)\right]^{\mathrm{T}}$ of the unstable mode are shown in Fig. 2C. Also, the dynamics of the unstable base flow pattern are presented in Fig. 2D. The period of the unstable mode is $37 \mathrm{~min}$, and thus its oscillation is relatively slow. The spatial shape of the mode indicates that there are four large magnitude countries involved. The computation shown in Fig. $2 \mathrm{~B}$ reflects the Koopman eigenvalues obtained during the computation over all 41 samples. It is of interest to consider the evolution of the eigenvalue computation over time, to understand the persistence of the unstable KM in relatively noisy data. We computed the Koopman eigenvalues (and the associated KMs) starting from the 1st sample and computing until $N=8,9, \ldots, 40$, namely, for many sets of samples with different final number $N$. The result is shown in Fig. 3 as the number density plot, where the color changes from blue to red and depicts the number density of unstable Koopman eigenvalues in each cell: see Appendix C for details. In this figure, the red cells are close to the unstable Koopman eigenvalues shown in Fig. 2. Therefore, the unstable KM persists under a change of the sample length. As reviewed above, the dynamics of power exchange deviations are closely related to the initiation of the system disturbance. The base flow pattern of the unstable KM presented in Fig. 2D vividly shows how the large-scale unstable dynamics can be extracted from the noisy data-the signature of the instability, in other words, the breaking of the $\mathrm{N}-1$ criterion is clearly present in

\footnotetext{
${ }^{1}$ The $\mathrm{N}-1$ criterion is a basic principle in power system operation [11]. This rule is that any single loss of transmission or generation element should not jeopardize the secure operation of the interconnected network, that is, trigger a cascade of line trippings or the loss of a significant amount of consumption.

${ }^{2}$ Since the number of samples $(N=41)$ is larger than the number of measurement site $(m=8)$, it is not possible to determine the unique KM decomposition: see [29] and Appendix A for details. To avoid this, here the decomposition by using the Moore-Penrose pseudo-inverse matrix is performed. In [29] it is demonstrated that the above technique of KM decomposition offers a result similar to that obtained via another technique based on Fourier-like formula [22].
} 

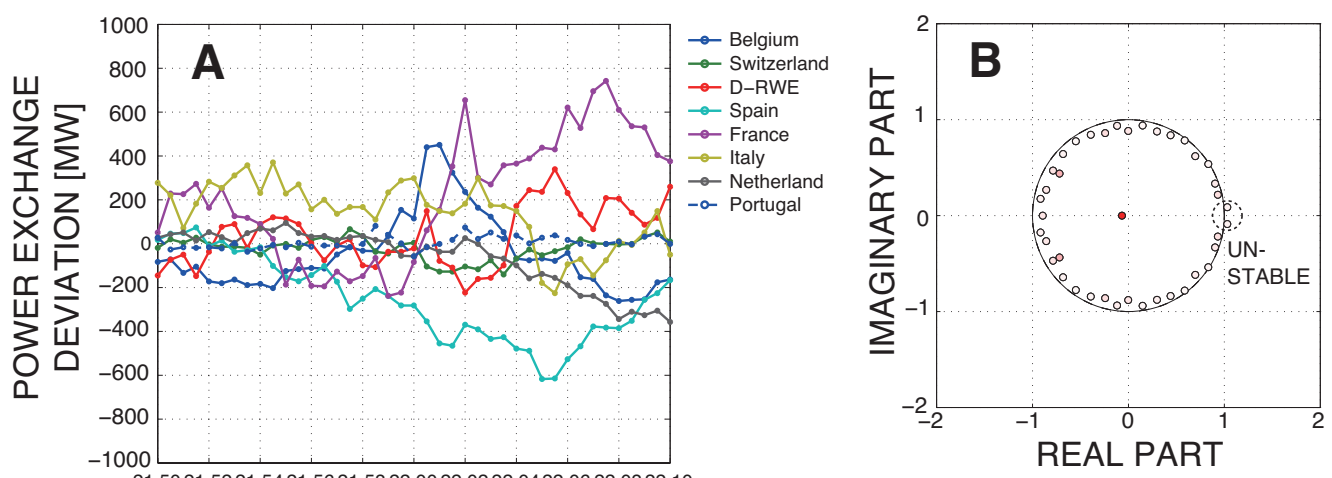

21:5021:5221:5421:5621:58 22:00 22:0222:0422:06 22:0822:10

REAL PART TIME (h:m)
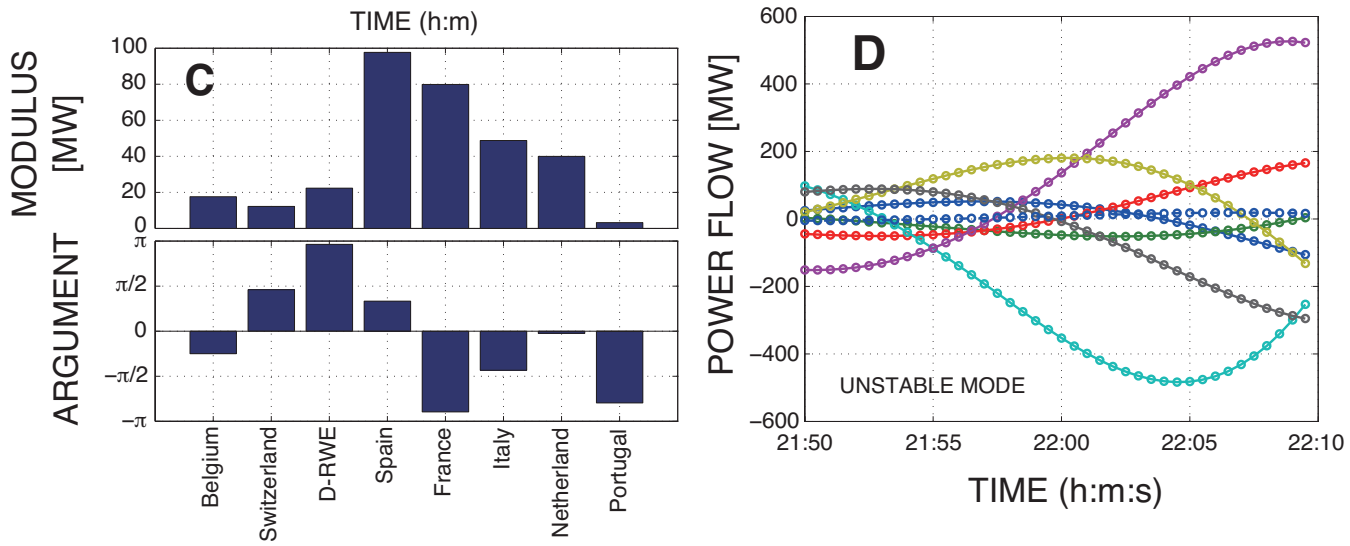

Fig. 2. Koopman mode analysis of power exchange deviations leading to the 2006 system disturbance in the European grid: (A) data on power exchange deviations; (B) Koopman eigenvalues $\tilde{\lambda}_{j}$; (C) modulus and argument vectors of unstable Koopman mode; and (D) dynamics of base flow patterns (9) for the unstable mode. A power exchange deviation is the difference between the actual power exchanges and the scheduled power exchanges with other countries.

the dynamics even before time 22:00. Recall that the UCTE report argues that the situation is rather normal and typical at that time.

\section{Conclusions}

It is often difficult to unravel large trends in complex cascading instability events in power systems. In this paper, we have developed a data-based approach to stability assessment of power systems that does not require any development of models. This approach was applied to analyze the data on physical power flows recorded in two major accidents: the 2011 Arizona-Southern California grid outage and the 2006 system disturbance in the European grid. We showed that Koopman modes associated with unstable Koopman eigenvalues account for the spatial dynamics of coherent power flows that are involved in these accidents. The KMA enables the assessment of stability without models, in a fully nonlinear and-one could argue-chaotic situation, where many operator actions are taken in response to the unraveling cascading failure. It is important to note that these actions, evidenced in the reports [11] and [34], would be extremely difficult to model due to their discrete and/or stochastic nature. Yet, the KMA seems to capture the main coherent, global, unstable trends accurately, starting from a simple dataset. We speculate that, based on recent developments of PMU's and thus a qualitative and quantitative improvement of available data, control mechanisms accounting for global KM features deduced from data can serve as a basis for system-wide stabilization.
Another use of these sorts of data analysis is in situational awareness. Two main causes of the 2011 Arizona-Southern California grid outage are stated in the official report [34]: lack of adequate situational awareness and lack of coordination of protection systems. Also, in the 2006 system disturbance of the European grid [11], the lack of online security computation (analysis of N-1 criterion) based on real-time data on the grid's state is indicated. System-level indicators based on KMA could provide system operators with coarse-grained information on global unstable trends. This would enable preventive global measures for containment of cascading outages and reduction of the incidence of wide-area blackouts.

As mentioned above and in [8], the detailed dynamics in cascading failures are modeled by a hybrid dynamical system with both continuous- and discrete-valued variables. This is based on the so-called microscopic description of the power system dynamics that uses detailed mathematical models of circuits and apparatuses. On the other hand, macroscopic description of the dynamics is expected to describe large trends of power systems and a signature of system-wide instabilities. Here, active power flows are regarded as one of macroscopic variables for power systems and are expected to exhibit relatively-continuous and slow changes (see Section III). That is why in this paper the time evolution of the active power flows is well captured by the Koopman operator theory. Applying the theory to non-smooth dynamics of phases and voltages in cascading failures is an interesting direction that we hope to pursue in the future. Also, extending the theory to 


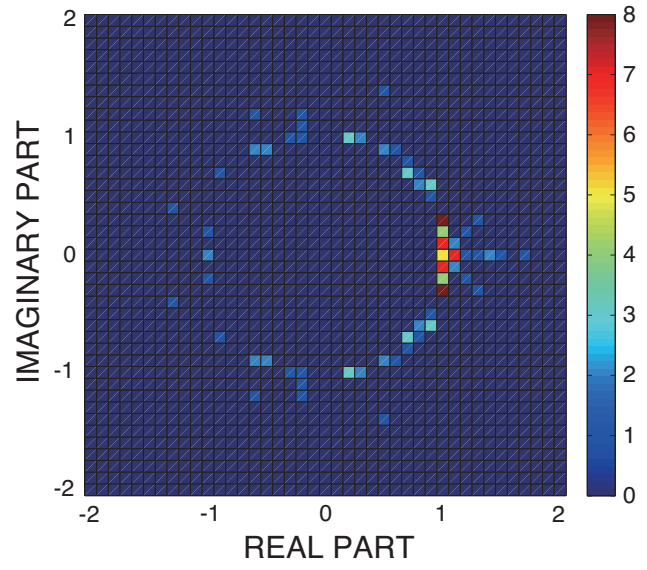

Fig. 3. Number density of unstable Koopman eigenvalues for power exchange deviations leading to the 2006 system disturbance in the European grid

non-smooth and hybrid cases is one of our future directions. It is interesting to note that Koopman operator theory often does not require smoothness (or even continuity) of the underlying dynamics.

\section{ACKNOWLEDGEMENTS}

We would like to thank Professor Takashi Hikihara of Kyoto University for encouragement and constant support of our work. We are also grateful to Dr. Sophie Loire for her careful reading of the manuscript and to anonymous reviewers for their constructive comments.

\section{APPENDIX A \\ Computation of KoOpMan Modes}

Here, the Arnoldi-like algorithm for computation of Koopman eigenvalues and Koopman modes [23] is introduced. For a given set of finite-time data described by (1), the empirical Ritz values $\tilde{\lambda}_{j}$ and empirical Ritz vectors $\tilde{\boldsymbol{V}}_{j}$ of this data are defined with the following algorithm:

(i) Define constants $c_{j}$ such that for vector $\boldsymbol{r}$ satisfying $\boldsymbol{r} \perp \operatorname{span}\left\{\boldsymbol{P}_{0}, \boldsymbol{P}_{1}, \ldots, \boldsymbol{P}_{N-2}\right\}$,

$$
\boldsymbol{r}=\boldsymbol{P}_{N-1}-\sum_{j=0}^{N-2} c_{j} \boldsymbol{P}_{j}
$$

(ii) Define the companion matrix $\mathrm{C}$ as

$$
\mathrm{C}:=\left[\begin{array}{ccccc}
0 & 0 & \cdots & 0 & c_{0} \\
1 & 0 & \cdots & 0 & c_{1} \\
0 & 1 & \cdots & 0 & c_{2} \\
\vdots & \vdots & \ddots & \vdots & \vdots \\
0 & 0 & \cdots & 1 & c_{N-2}
\end{array}\right]
$$

and find its $N-1$ eigenvalues $\tilde{\lambda}_{1}, \ldots, \tilde{\lambda}_{N-1}$.

(iii) Define the Vandermonde matrix $\mathrm{T}$ using $\tilde{\lambda}_{j}$ as

$$
\mathrm{T}:=\left[\begin{array}{ccccc}
1 & \tilde{\lambda}_{1} & \tilde{\lambda}_{1}^{2} & \cdots & \tilde{\lambda}_{1}^{N-2} \\
1 & \tilde{\lambda}_{2} & \tilde{\lambda}_{2}^{2} & \cdots & \tilde{\lambda}_{2}^{N-2} \\
\vdots & \vdots & \vdots & \ddots & \vdots \\
1 & \tilde{\lambda}_{N-1} & \tilde{\lambda}_{N-1}^{2} & \cdots & \tilde{\lambda}_{N-1}^{N-2}
\end{array}\right]
$$

(iv) Define $\tilde{\boldsymbol{V}}_{j}$ to be the columns of $\mathrm{V}:=$ $\left[\begin{array}{l}\boldsymbol{P}_{0} \boldsymbol{P}_{1} \cdots \boldsymbol{P}_{N-2}\end{array}\right] \mathrm{T}^{-1}$.

Then, we have the following equations that are originally derived in [23]:

$$
\boldsymbol{P}_{k}=\sum_{j=1}^{N-1} \tilde{\lambda}_{j}^{k} \tilde{\boldsymbol{V}}_{j}, \quad \boldsymbol{P}_{N-1}=\sum_{j=1}^{N-1} \tilde{\lambda}_{j}^{N-1} \tilde{\boldsymbol{V}}_{j}+\boldsymbol{r},
$$

where $k=0,1, \ldots, N-2$. Comparing with (8), the empirical Ritz values $\tilde{\lambda}_{j}$ and vectors $\tilde{\boldsymbol{V}}_{j}$ behave precisely in the same manner as the Koopman eigenvalues $\lambda_{i}$ and the terms $\varphi_{i}\left(\boldsymbol{x}_{0}\right) \boldsymbol{V}_{i}$ containing Koopman eigenfunctions and KMs, but for the finite sum (13) instead of the infinite sum (8).

Here, it is valuable to discuss how to compute the constant vector $\boldsymbol{c}:=\left(c_{0}, c_{1}, \ldots, c_{N-2}\right)^{\mathrm{T}} \in \mathbb{R}^{N-1}$ from the data (1). Because of $\boldsymbol{r} \perp \operatorname{span}\left\{\boldsymbol{P}_{0}, \boldsymbol{P}_{1}, \ldots, \boldsymbol{P}_{N-2}\right\}$, by multiplying $\boldsymbol{P}_{i}^{\mathrm{T}}(i=0,1, \ldots, N-2)$ on both sides of (10) we have

$$
0=\boldsymbol{P}_{i}^{\mathrm{T}} \boldsymbol{P}_{N-1}-\sum_{j=0}^{N-2} c_{i} \boldsymbol{P}_{i}^{\mathrm{T}} \boldsymbol{P}_{j}
$$

Now let us define the matrix $\mathrm{A}=\left\{A_{i j}\right\} \in \mathbb{R}^{(N-1) \times(N-1)}$ as $A_{i j}=\boldsymbol{P}_{i}^{\mathrm{T}} \boldsymbol{P}_{j}$ and the vector $\boldsymbol{b} \in \mathbb{R}^{N-1}$ as $b_{i}=\boldsymbol{P}_{i}^{\mathrm{T}} \boldsymbol{P}_{N-1}$. Then, we have

$$
\mathbf{0}=\boldsymbol{b}-\mathrm{A} \boldsymbol{c} .
$$

If $A$ is full-rank, then there exists the unique solution $c$ for (15). This case appears in analysis of the 2011 ArizonaSouthern California grid outage, where the number of samples $N$ is equal to 9 and the dimension $m$ of $\boldsymbol{P}_{k}$ is equal to 8 , namely $N-1=m$. If A is not full-rank, there exists no unique solution of (15). This case appears in analysis of the 2006 system disturbance in the UCTE grid, where $N-1>m$ and the rank of $A$ is at most $m$. In this case, we used a solution to minimize the norm $\|\boldsymbol{b}-A \boldsymbol{c}\|$ using the Moore-Penrose pseudo-inverse matrix of $A$, denoted by $A^{\dagger}$. This matrix is computed with the function pinv in MATLAB. The solution corresponds to $\mathrm{A}^{\dagger} \boldsymbol{b}$.

\section{APPENDIX B \\ One-Line Diagram of The SAN Diego AREA POWER SYSTEM}

A schematic one-line diagram of the San Diego area power system is presented in Fig. 4 to show the location where the data were collected.

\section{APPENDIX C \\ Computation of Eigenvalue Density}

For the analysis of the 2006 system disturbance in the UCTE grid, the number density of unstable Koopman eigenvalues for many sets of samples with different final number $N$ was shown. Here we describe how to plot the number density of unstable eigenvalues. For each $N=8,9, \ldots, 40$, the Koopman eigenvalues and associated KMs are computed. We select a domain of complex plane around the origin, defined as $[-2-$ $h / 2,2+h / 2] \times[-2-h / 2,2+h / 2]$ with $h=1 / 10$, and divide it to a union of (square) cells with length $h$. Then, for each unstable Koopman eigenvalue we choose a single cell 


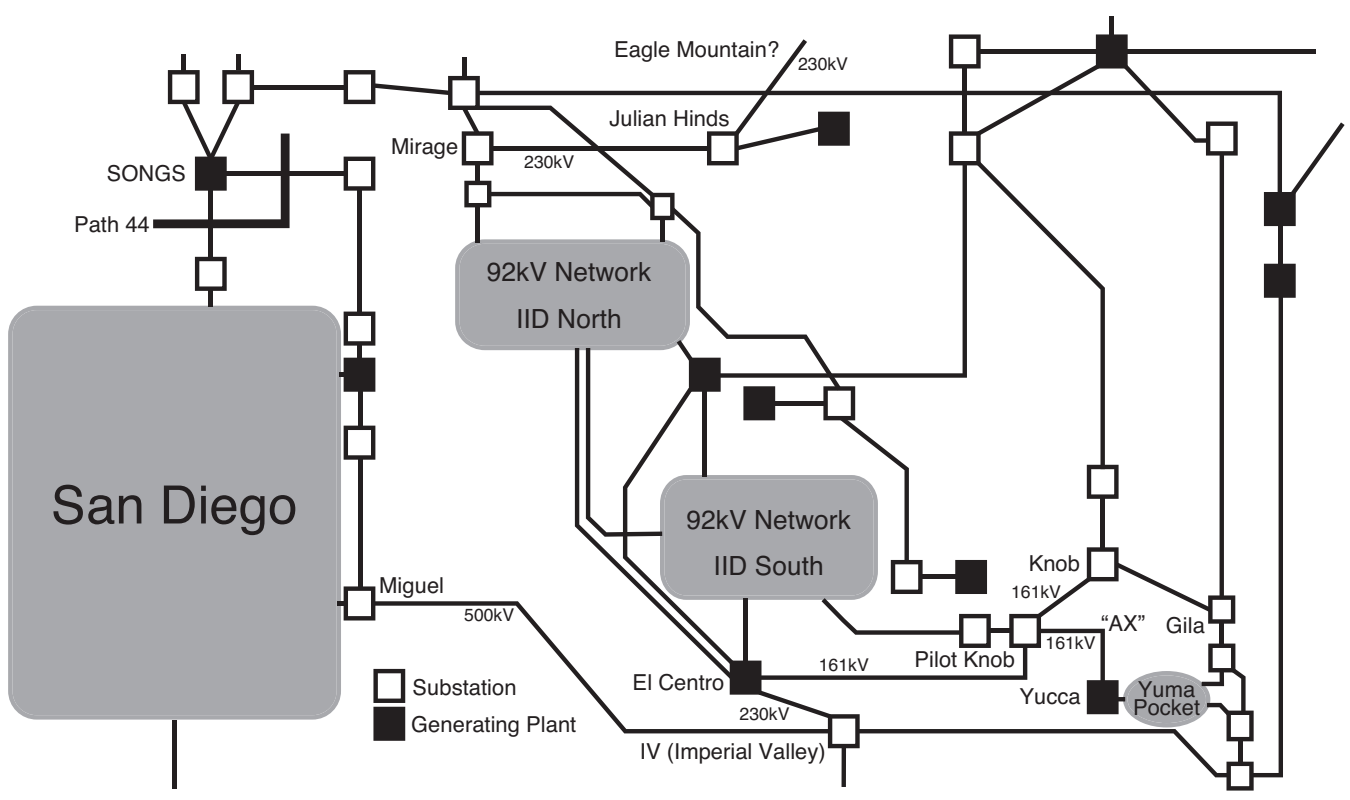

Fig. 4. Schematic one-line diagram of the San Diego area power system. This is based on the figure in page 4 of [34]. Voltage levels of lines, location of transformers, and multiple parallel lines are omitted.

containing it and count the number of eigenvalues in each cell. By repeating this process for all $N$, the number density of unstable Koopman eigenvalues is computed.

\section{REFERENCES}

[1] G. Andersson, P. Donalek, R. Farmer, N. Hatziargyriou, I. Kamwa, P. Kundur, N. Martins, J. Paserba, P. Pourbeik, J. Sanchez-Gasca, R. Schulz, A. Stankovic, C. Taylor, and V. Vittal, "Causes of the 2003 major grid blackouts in North America and Europe, and recommended means to improve system dynamic performance," IEEE Trans. Power Syst., vol. 20, no. 4, pp. 1922-1928, November 2005.

[2] C. W. Taylor and D. C. Erickson, "Recording and analyzing the July 2 cascading outage," IEEE Comput. Appl. Power, vol. 10, no. 1, pp. 26-30, 1997.

[3] D. N. Kosterev, C. W. Taylor, and W. A. Mittelstadt, "Model validation for the August 10, 1996 WSCC system outage," IEEE Trans. Power Syst., vol. 14, no. 3, pp. 967-979, August 1999.

[4] I. A. Hiskens and M. Akke, "Analyis of the Nordel power grid disturbance of January 1, 1997 using trajectory sensitivities," IEEE Trans. Power Syst., vol. 14, no. 3, pp. 987-994, August 1999.

[5] V. M. Venkatasubramanian and Y. Li, "Analysis of 1996 Western American electric blackouts," in Proc. Bulk Power System Dynamics and Control-VI, Cortina d'Ampezzo, Italy, August 2004, pp. 685-721.

[6] R. Kinney, P. Crucitti, R. Albert, and V. Latora, "Modeling cascading failures in the North American power grid," Eur. Phys. J. B, vol. 46, pp. 101-107, 2005.

[7] I. Dobson, B. A. Carreras, V. E. Lynch, and D. E. Newman, "Complex systems analysis of series of blackouts: Cascading failure, critical points, and self-organization," CHAOS, vol. 17, no. 2, p. 02610, June 2007.

[8] Y. Susuki, Y. Takatsuji, and T. Hikihara, "Hybrid model for cascading outage in a power system: A numerical study," IEICE Trans. Fund. Electr., vol. E92-A, no. 3, pp. 871-879, March 2009.

[9] Prepared by the IEEE PES CAMS Task Force on Understanding, Predicition and Restoration of Cascading Failures, 2008, Initial review of methods for cascading failure analysis in electric power transmission systems, Proc. IEEE PES General Meeting (Pittsburgh, USA).

[10] D. Gautam, V. Vittal, and T. Harbour, "Impact of increased penetration of DFIG-based wind turbine generators on transient and small signal stability of power systems," IEEE Trans. Power Syst., vol. 24, no. 3, pp. 1426-1434, August 2009.

[11] Issued by Union for the Co-ordination of Transmission of Electricity, "Final Report, System Disturbance on 4 November 2006," 2007.

[12] E. W. Kimbark, Power System Stability. New York: John Wiley \& Sons, 1947, vol. I.
[13] J. Machowski, J. W. Bialek, and J. R. Bumby, Power System Dynamics and Stability. England: John Wiley \& Sons, 1997.

[14] H. D. Chiang, "Power system stability" in Wiley Encyclopedia of Electrical and Electronics Engineering, J. G. Webster, Ed. New York: John Wiley \& Sons, March 1999, pp. 105-137.

[15] A. G. Phadke, "Synchronized phasor measurement in power systems," IEEE Comput. Appl. Power, vol. 6, no. 2, pp. 10-15, April 1993.

[16] J. De La Ree, V. Centeno, J. S. Thorp, and A. G. Phadke, "Synchronized phasor measurement applications in power systems," IEEE Trans. Smart Grid, vol. 1, no. 1, pp. 20-27, June 2010.

[17] A. Armenia and J. H. Chow, "A flexible phasor data concentrator design leveraging existing software technologies," IEEE Trans. Smart Grid, vol. 1, no. 1, pp. 73-81, June 2010.

[18] D. R. Ostojić, "Spectral monitoring of power system dynamic performances," IEEE Trans. Power Syst., vol. 8, no. 2, pp. 445-451, May 1993.

[19] A. R. Messina and V. Vittal, "Nonlinear, non-stationary analysis of interarea oscillations via Hilbert spectral analysis," IEEE Trans. Power Syst., vol. 21, no. 3, pp. 1234-1241, August 2006.

[20] _ - "Extraction of dynamic patterns from wide-area measurements using empirical orthogonal functions," IEEE Trans. Power Syst., vol. 22, no. 2, pp. 682-692, May 2007.

[21] I. Mezić and A. Banaszuk, "Comparison of systems with complex behavior," Physica D, vol. 197, pp. 101-133, 2004.

[22] I. Mezić, "Spectral properties of dynamical systems, model reduction and decompositions," Nonlinear Dyn., vol. 41, pp. 309-325, August 2005.

[23] C. W. Rowley, I. Mezić, S. Bagheri, P. Schlatter, and D. S. Henningson, "Spectral analysis of nonlinear flows," J. Fluid Mech., vol. 641, pp. 115-127, 2009.

[24] M. Budišić, R. Mohr, and I. Mezić, "Applied Koopmanism," CHAOS, vol. 22, p. 047510, 2012.

[25] I. Mezić, "Analysis of fluid flows via spectral properties of Koopman operator," Аппи. Rev. Fluid Mech., vol. 45, pp. 357-378, 2013.

[26] B. O. Koopman, "Hamiltonian systems and transformations in Hilbert space," Proc. Natl. Acad. Sci. USA, vol. 17, no. 5, pp. 315-318, May 1931.

[27] K. Peterson, Ergodic Theory. Cambridge: Cambridge University Press, 1983.

[28] A. Lasota and M. C. Mackey, Chaos, Fractals, and Noise: Stochastic Aspects of Dynamics. New York: Springer-Verlag, 1994.

[29] Y. Susuki and I. Mezić, "Nonlinear Koopman modes and coherency identification of coupled swing dynamics," IEEE Trans. Power Syst., vol. 26, no. 4, pp. 1894-1904, November 2011, (also, correction, this journal, vol. 26, no. 4, p. 2584, November 2011). 
[30] - "Nonlinear Koopman modes and a precursor to power system swing instabilities," IEEE Trans. Power Syst., vol. 27, no. 3, pp. 1182 1191, March 2012.

[31] B. Eisenhower, T. Maile, M. Fischer, and I. Mezić, "Decomposing building system data for model validation and analysis using the Koopman operator," in Proc. IBPSA Simbuild Conference, New York City, USA, August 2010, pp. 434-441.

[32] F. B. Hildebrand, Introduction to Numerical Analysis. New York: McGraw-Hill, 1956

[33] J. F. Hauer, C. J. Demeure, and L. L. Scharf, "Initial results in Prony analysis of power system response signals," IEEE Trans. Power Syst. vol. 5, no. 1, pp. 80-89, February 1990.

[34] Prepared by the Staffs of the Federal Energy Regulatory Commission and the North American Electric Reliability Corporation, "ArizonaSouthern California Outages on September 8, 2011, Causes and Recommendations," 2012

Yoshihiko Susuki (S'01-M'05) received the bachelor, master, and Ph.D degrees in engineering from Kyoto University, Kyoto, Japan, in 2000, 2002, and 2005 , respectively.

In 2005, he joined the Department of Electrical Engineering, Kyoto University, where he is currently a Lecturer. In 2008-2010, he was a Visiting Researcher in the Department of Mechanical Engineering, University of California, Santa Barbara, CA, under JSPS Postdoctoral Fellowship for Research Abroad. His research interests are in applied nonlinear dynamics, power and energy technology, and control applications.

Igor Mezic (M'08) received the Ph.D. degree in applied mechanics from California Institute of Technology, Pasadena, CA, in 1994.

$\mathrm{He}$ is currently a Professor of the Department of Mechanical Engineering, and the Director of Center for Energy-Efficient Design and the Head of Buildings and Design Solutions Group of the Institute of Energy Efficiency at the University of California, Santa Barbara, CA. 